\title{
Crystallization Processes of Amorphous GeSn Thin Films by Heat Treatment and Electron Beam Irradiation
}

\author{
T. Kimura ${ }^{1}$, M. Ishimaru ${ }^{1}$, M. Okugawa ${ }^{2}$, R. Nakamura ${ }^{2}$ and H. Yasuda ${ }^{3}$ \\ 1. Department of Materials Science and Engineering, Kyushu Institute of Technology, Kitakyushu, \\ Fukuoka 804-8550, Japan \\ 2. Department of Materials Science, Osaka Prefecture University, Sakai, Osaka 599-8531, Japan \\ 3. Research Center for Ultra-High Voltage Electron Microscopy, Osaka University, Ibaraki, Osaka \\ 567-0047, Japan
}

Germanium-tin (GeSn) is anticipated as a component of high-efficiency thin film solar cells, because of its tunable band gap and lattice parameter by changing Sn concentration [1]. For the application, it is necessary to control the Sn concentration in wide range. However, the solubility limit of Sn in Ge matrix is very small, and therefore Sn segregation easily occurs during heat treatment. Recrystallization from amorphous is one of the possible ways to enhance Sn concentration. In fact, GeSn with 25 at $\% \mathrm{Sn}$ was obtained by thermal annealing at $70^{\circ} \mathrm{C}$ [2]. Low temperature annealing can suppress diffusion of Sn. The synthesis of materials using radiation fields is useful to realize nonequilibrium materials at room temperature. The purpose of this study is to examine crystallization process of amorphous GeSn under heat treatment and electron beam irradiation by transmission electron microscopy (TEM).

Amorphous GeSn thin films were prepared by radio frequency magnetron sputtering method. The Sn chip was placed on the Ge target, and the Sn concentration was controlled by changing the number of chips. Figure 1 shows the sample preparation procedure for TEM observations. The thin film was deposited on a $\mathrm{NaCl}$ substrate. The substrate was dissolved by water, and the separated thin film was scooped with a Mo grid. Structural changes during heat treatment and electron beam irradiation were examined by in situ TEM observations.

Figure 2 shows a high-resolution TEM (HRTEM) image and the corresponding electron diffraction patterns of as-sputtered specimen prepared by placing $30 \mathrm{Sn}$ chips on a Ge target. The HRTEM image reveals a typical salt and pepper contrast and halo rings appear in the diffraction pattern, indicating that this specimen is amorphous and no remarkable phase separation occurs.

Figure 3 shows electron diffraction patterns as a function of annealing temperature: (a-c) heating process and (d-f) cooling one. From the EDX analysis, the Sn concentration was estimated to 9.7 at $\%$. The amorphous structure was maintained up to $375^{\circ} \mathrm{C}$, but it was found that crystallization started at $400{ }^{\circ} \mathrm{C}$ (Fig. 3(b)). Finally, complete recrystallization occurs at $500{ }^{\circ} \mathrm{C}$. It should be noted that only the ring pattern due to the diamond structure appears in Fig. 3(c). This feature remains at $150{ }^{\circ} \mathrm{C}$ during cooling process (Fig. 3(d)), while addition reflections due to $\beta$-Sn appear at $100{ }^{\circ} \mathrm{C}$ (Fig. 3(e)). The precipitation temperature of $\mathrm{Sn}$ observed here is much lower than the melting point of $\mathrm{Sn}\left(\sim 230^{\circ} \mathrm{C}\right)$. It is reported that the melting point depression occurs depending on particle size [3]. Based on this result, we think the melting point depression is due to the formation of nano-sized $\mathrm{Sn}$ particles.

Figure 4 shows the bright field image and the electron diffraction pattern of the specimen with 20.1 at\% Sn after electron-beam irradiation. The specimen was irradiated with $125 \mathrm{keV}$ electrons at a flux of $0.15 \mathrm{nA} / \mathrm{sec}$ for $3 \mathrm{~min}$ at room temperature. It was found that the specimen is completely recrystallized. 
In the diffraction pattern, only the Debye-Scherrer rings of the diamond structure was observed. Although the Sn concentration is higher than the heat treated sample (Fig. 3), the Sn precipitation is suppressed in this specimen. This suggests that electron beam irradiation can realize crystalline Ge with high concentration $\mathrm{Sn}$ at room temperature. The structural changes of materials under radiation environment are induced by two mechanisms: one is knock-on effect and the other is electron excitation one. When the energy of incident electrons is larger than the displacement energy of the target material, the knock-on effect becomes more pronounced. The knock-on energy, $E_{\max }$, is equal to $4.2 \mathrm{eV}$ under the present irradiator conditions (i.e., $E=125 \mathrm{kV}$ ). This value is smaller than the displacement energy required for the crystallization of amorphous $\mathrm{Ge}(7.2 \mathrm{eV})[4]$, and therefore crystallization mechanism in the present study is attributed to the electron excitation effect.

In conclusion, the thermally-induced and electron beam irradiation-induced crystallization of amorphous GeSn was investigated by in situ TEM. Sn segregation occurred in 9.7 at\% Sn specimen during heat treatment, whereas no Sn segregation was observed in 20.1 at $\%$ Sn specimen crystallized by electron beam irradiation at room temperature. This suggests that electron-beam-irradiation techniques is useful for realizing the low-temperature synthesis of crystalline Ge with high concentration Sn.

\section{References:}

[1] S. Zaima et al., Sci. Technol. Adv. Mater. 16 (2015) 043502.

[2] K. Toko et al., Appl. Phys. Lett. 106 (2015) 082109.

[3] J.-G. Lee and H. Mori, Thin Solid Films 546 (2013) 453.

[4] I. Jencic, I.M. Robertson and J. Skvarc, Nucl. Instr. Meth. Phys. Res. B 148 (1999) 345.

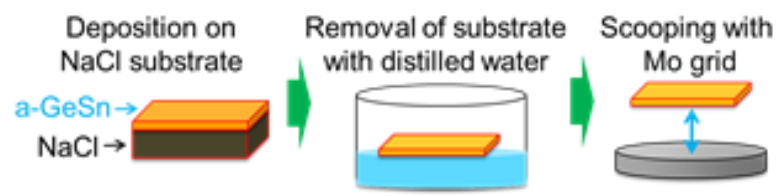

Figure 1. Sample preparation procedure for TEM observations.

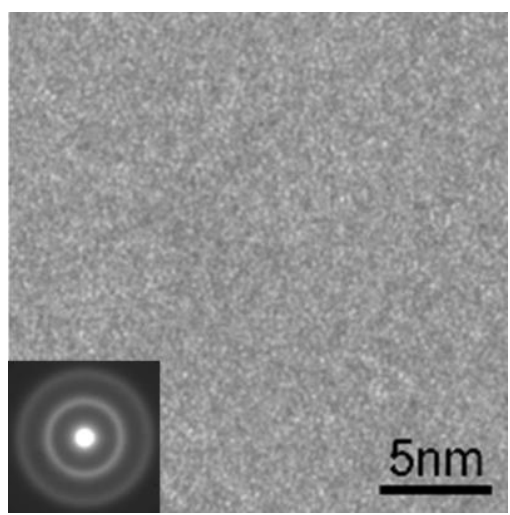

Figure 2. HRTEM image and the corresponding electron diffraction pattern of as-sputtered specimen with 20.1 at $\% \mathrm{Sn}$.
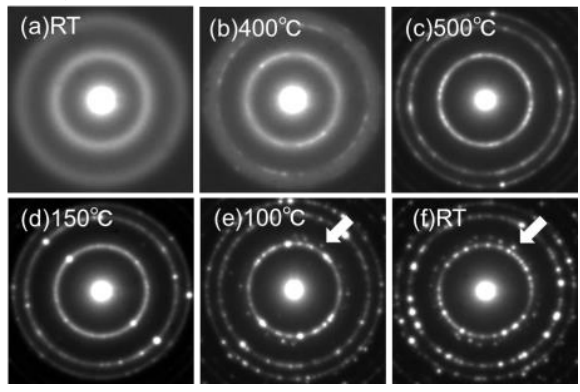

Figure 3. Electron diffraction patterns of 9.7 at $\%$ Sn specimen during heat treatment.

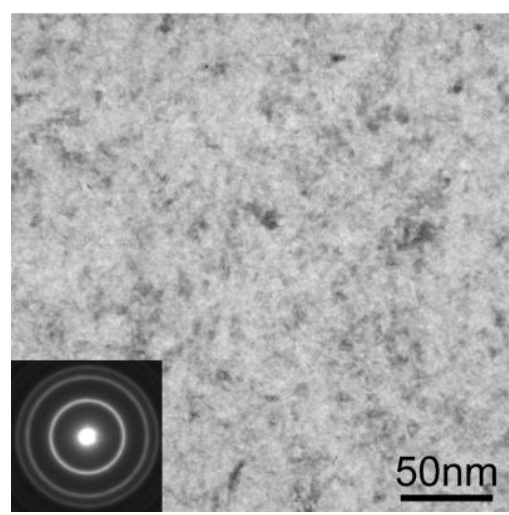

Figure 4. Bright field image and the electron diffraction pattern of $20.1 \mathrm{at} \% \mathrm{Sn}$ specimen after irradiation. 